\title{
Relação entre níveis de chumbo no colostro, consumo alimentar e características socioeconômicas de puérperas em Goiânia, Brasil
}

\author{
Renata Moreira Gonçalves, ${ }^{1}$ José Rubens Gonçalves ${ }^{2}$ \\ e Nélida Schmid Fornés ${ }^{3}$
}

Como citar Gonçalves RM, Gonçalves JR, Fornés NS. Relação entre níveis de chumbo no colostro, consumo alimentar e características socioeconômicas de puérperas em Goiânia, Brasil. Rev Panam Salud Publica. 2011:29(4):227-33.

RESUMO Objetivo. Estimar a concentração de chumbo em colostro humano e verificar a existência de relação entre esse dado, consumo alimentar e fatores socioeconômicos das puérperas.

Métodos. Estudo transversal com 80 puérperas de uma maternidade de Goiânia, Brasil, realizado de julho a dezembro de 2008. Aplicou-se um questionário socioeconômico e de estilo de vida, além do questionário de frequência de consumo alimentar. Posteriormente, foram coletadas amostras de colostro, analisadas por espectrometria de absorção atômica por chama para quantificar o chumbo. Calculou-se a correlação entre o teor de chumbo, as variáveis socioeconômicas e o consumo alimentar.

Resultados. A média da concentração de chumbo foi $6,88 \mu \mathrm{g} / \mathrm{L}$, e a mediana foi 4,65 $\mu \mathrm{g} / \mathrm{L}$. Os aspectos socioeconômicos não apresentaram associação significativa com o teor de chumbo. A ingestão de ácidos graxos saturados, ovos, carnes suinas, frango e derivados (todos com valores de $r=-0,26$ e $\mathrm{P}=0,020$ ) correlacionou-se inversamente ao teor chumbo do colostro humano, ao passo que a ingestão de abóbora $(r=0,26$ e $\mathrm{P}=0,019)$ e berinjela $(r=0,27$ e $\mathrm{P}=0,015)$ correlacionou-se proporcionalmente ao valor obtido. A correlação entre abóbora cozida, ovos, carnes suínas, de frango e derivados foi mantida após ajuste na regressão logística. Conclusões. Foi detectada presença de chumbo no colostro materno, possivelmente como resultado da exposição dietética das puérperas deste estudo. Além da correlação entre presença de chumbo e consumo alimentar, os resultados sugerem que a ingestão de nutrientes específicos pode propiciar maior ou menor concentração desse contaminante, sem relação direta com fatores socioeconômicos.

Palavras-chave Chumbo; consumo de alimentos; colostro; leite humano; toxicidade; Brasil.

1 Universidade Federal de Goiás (UFG), Faculdade de Medicina, Programa de Pós-Graduação em Ciências da Saúde, Goiânia (GO), Brasil; e Faculdade Anhanguera de Anápolis, Anápolis (GO), Brasil. Correspondência: renata.nut@gmail.com

2 Ministério da Agricultura, Pecuária e Abastecimento (MAPA) e Faculdade de Tecnologia SENAI Roberto Mange, Goiânia (GO), Brasil.

3 UFG, Faculdade de Nutrição, Goiânia (GO), Brasil.
O leite materno é a melhor forma de alimentar o recém-nascido, sendo recomendado como alimento exclusivo até os 6 meses de vida por conter os nutrientes necessários ao organismo infantil. Entretanto, além de nutrientes e minerais essenciais para o adequado cresci- mento da criança, o leite materno pode transportar metais pesados, que podem ser tóxicos (1), tornando-se uma forma de exposição nessa fase de vida.

De maneira geral, os lactentes fazem parte da população mais suscetível ao chumbo, pois apresentam maior ab- 
sorção gastrintestinal e excreção renal menos efetiva, além de uma barreira hematoencefálica menor se comparados aos adultos. A vulnerabilidade ao chumbo depende do estágio de desenvolvimento em que a criança se encontra (existem períodos críticos de desenvolvimento estrutural e funcional, tanto no estágio prénatal quanto no pós-natal, em que uma estrutura ou função específica poderá ser mais sensível a danos), da dose, da duração da exposição e do estado nutricional da criança (2). Assim, é importante determinar a presença desse metal pesado no leite materno, e o colostro é considerado um biomarcador adequado para esse fim.

Como não existe uma concentração de chumbo em colostro humano que seja considerada como inofensiva, a exposição ambiental, com consequente introdução do metal no organismo, até mesmo em baixos níveis, é um problema de saúde pública, tendo em vista a habilidade do chumbo de se acumular no corpo humano por um longo período de tempo $(3,4)$. O contato com o chumbo desde o início da vida pode ter efeitos desastrosos, especialmente nos sistemas nervoso e hematopoiético, com influência no crescimento e no desenvolvimento mental do neonato. Esses problemas cognitivos e comportamentais podem ter um impacto mais tardio, na fase escolar da criança (4). A alimentação adequada da gestante pode ser um fator-chave para a redução do risco de exposição ao chumbo. Além disso, a suplementação nutricional da dieta materna modifica o processo de absorção, transporte, armazenamento e inativação de chumbo, reduzindo sua toxicidade. Isso demonstra que uma alimentação equilibrada em nutrientes essenciais pode reduzir, ou até mesmo impedir, a absorção do metal chumbo (5).

Considerando esses aspectos, conduziu-se este estudo, cujo objetivo foi investigar a concentração de chumbo no colostro humano e verificar a relação entre essa concentração, o consumo alimentar e o status socioeconômico de puérperas.

\section{MATERIAIS E MÉTODOS}

O presente estudo transversal descritivo foi realizado de julho a dezembro de 2008 na Maternidade Nossa Senhora de Lourdes, entidade pública localizada na Cidade de Goiânia, Brasil. A amostra de conveniência incluiu 80 puérperas que preencheram os seguintes critérios de inclusão: idade igual ou superior a 18 anos; ser sadias e sem doenças crônicas; gravidez desenvolvida sem intercorrências, com parto normal; estar no período de até 5 dias pós-parto; ter tido o recémnascido a termo; facilidade para fornecer o colostro do seio. Foram utilizados os seguintes critérios de exclusão: problemas ou doenças durante o período da gestação e no parto; ter tido o bebê prétermo; ser portadora de necessidades especiais que exigissem atendimento especial de saúde; e não ter produzido colostro suficiente ou ter dificuldades para ejetar leite para alimentar o filho.

Todas as mães que preenchiam os critérios de inclusão concordaram em participar após terem sido informadas com relação ao tipo de estudo e aos procedimentos realizados. As participantes assinaram um termo de consentimento livre e esclarecido. $\mathrm{O}$ projeto foi submetido e aprovado pelo Comitê de Ética em Pesquisa da Universidade Federal de Goiás.

Para a coleta dos dados, realizada por entrevista direta, utilizou-se um questionário padronizado. Foram coletados dados socioeconômicos, demográficos e de estilo de vida, como idade, renda, escolaridade, ocupação, região da residência (próxima ou não de locais considerados contaminados, ou seja, indústrias, postos de gasolina, rios poluídos, casas de fundição, fábricas de baterias e cerâmica, entre outros) $(6,7)$, etilismo e hábito de fumar. Aplicou-se outro questionário para obter a frequência de consumo alimentar (QFCA) (8).

O QFCA é um método estruturado qualitativo e quantitativo que foi previamente validado e publicado (8) para populações de baixa renda, referindo-se à ingestão de alimentos nos últimos 6 meses de gestação. Possui 127 itens alimentares distribuídos em 12 grupos: leite e derivados, leguminosas, ovos e carnes, cereais e derivados, massas e salgadinhos, açúcares e doces, frutas, hortaliças, gorduras, temperos e condimentos, bebidas alcoólicas, bebidas não alcoólicas e infusos, com as respectivas categorias de frequência de consumo (9).

Concluídas as entrevistas, as amostras de colostro foram coletadas das puérperas ainda na maternidade, por meio de ordenha manual, após higienização da mama com água deionizada. Nesse procedimento foram utilizadas luvas descartáveis. As amostras, que correspon- diam a $10 \mathrm{~mL}$ de colostro, foram colocadas diretamente em frascos estéreis de polietileno etiquetados, previamente tratados (lavados com água destilada, água deionizada, solução de ácido nítrico a 5\% e novamente com água deionizada). As amostras foram transportadas em caixa isotérmica à temperatura de 3 a $4{ }^{\circ} \mathrm{C}$ até a chegada ao laboratório, onde foram imediatamente congeladas a $-20^{\circ} \mathrm{C}$ até o momento das análises.

As amostras de colostro foram homogeneizadas e analisadas em duplicata (10). As análises foram realizadas no laboratório de equipamentos especializados de análises físico-químicas do Laboratório Nacional Agropecuário do Ministério da Agricultura, Pecuária e Abastecimento, conforme a metodologia oficial do Instituto Adolfo Lutz (10). A técnica utilizada para determinar o metal foi a espectrometria de absorção atômica com chama, em equipamento da marca Perkin Elmer, modelo AAnalyst 200, equipado com lâmpada de descarga elétrica (System 2 Electrodeless Discharge Lamps). O limite de detecção adotado para a pesquisa foi de $0,0016 \mu \mathrm{g} / \mathrm{L}$.

As variáveis foram analisadas no programa Statistical Package for the Social Sciences (SPSS) versão 13.0. As variáveis categóricas foram expressas em frequências e as variáveis contínuas, em medidas de tendência central. $O$ teste de Kolmogorov-Smirnov foi usado para verificar a normalidade das variáveis contínuas. O gráfico box plot foi utilizado para visualizar a distribuição das concentrações de chumbo segundo quartis.

O teste de Mann-Whitney foi utilizado para avaliar diferenças entre dois grupos de variáveis não paramétricas, e o teste de Kruskal-Wallis para avaliar diferenças entre mais de dois grupos, com intervalo de confiança de 95\% (IC95\%). O coeficiente de correlação de Spearman foi aplicado para testar o grau de correlações entre as variáveis contínuas para dados com distribuição não normal. Visto que esse grupo de variáveis não possuía distribuição normal, foi aplicada a função logarítmica para que modelos de regressões lineares fossem conduzidos com as mesmas variáveis da correlação, no intuito de confirmar quais variáveis independentes explicam melhor a variável resposta. Foram ajustadas as regressões lineares, considerando de forma isolada cada conjunto de variáveis independentes. Não necessariamente todas as variáveis foram utilizadas na re- 
gressão ajustada, somente aquelas escolhidas pelo método de seleção em etapas (stepwise), que fornece o melhor modelo. Considerou-se um nível de significância estatística de 5,0\%.

\section{RESULTADOS}

Participaram do estudo 80 puérperas com média de idade de 22,8 anos, sendo que $70,0 \%$ se encontravam na faixa etária entre 18 e 25 anos. Quanto à escolaridade, a média foi de 11,11 anos de estudo, e $61,2 \%$ tinham completado o ensino médio. Do total, $62,5 \%$ trabalhavam no lar. Todas as outras participantes exerciam as funções de comerciante, secretária, serviços gerais ou estudante, sendo consideradas não expostas ocupacionalmente de acordo com definição de outro autor (7). A renda per capita média foi de $\mathrm{R} \$ 217,49$. A maioria das entrevistadas $(83,8 \%)$ residiam em uma região da

TABELA 1. Distribuição das puérperas de uma maternidade segundo características socioeconômicas e de estilo de vida, Goiânia (GO), Brasil, 2008

\begin{tabular}{|c|c|c|}
\hline Variável & No. & $\%$ \\
\hline \multicolumn{2}{|l|}{ Idade (anos) } & 100 \\
\hline$<20$ anos & 20 & 25,0 \\
\hline$\geq 20$ anos & 60 & 75,0 \\
\hline \multicolumn{3}{|l|}{ Escolaridade } \\
\hline Fundamental & 28 & 35,0 \\
\hline Médio e/ou técnico & 49 & 61,2 \\
\hline \multicolumn{3}{|l|}{ Ensino superior e } \\
\hline pós-graduação & 3 & 3,8 \\
\hline \multicolumn{3}{|l|}{ Ocupação } \\
\hline Do lar & 50 & 62,4 \\
\hline \multicolumn{2}{|l|}{ Estudante, secretária, } & 26,3 \\
\hline Serviços gerais e faxina & 9 & 11,3 \\
\hline \multicolumn{3}{|l|}{ Renda per capita (SMª) } \\
\hline$<1 / 4$ & 12 & 15,0 \\
\hline $1 / 4$ a $1 / 2$ & 40 & 50,0 \\
\hline $1 / 2$ a 1 & 20 & 25,0 \\
\hline 1 a 2 & 6 & 7,5 \\
\hline Não mencionaram & 2 & 2,5 \\
\hline \multicolumn{3}{|l|}{ Estado civil } \\
\hline Solteira & 29 & 36,3 \\
\hline Casada & 51 & 63,7 \\
\hline \multicolumn{3}{|l|}{ Cidade onde reside } \\
\hline Goiânia & 67 & 83,8 \\
\hline Interior de Goiás & 13 & 16,2 \\
\hline \multicolumn{3}{|l|}{ Morou na zona rural } \\
\hline Sim & 55 & 68,8 \\
\hline Não & 25 & 31,2 \\
\hline \multicolumn{3}{|l|}{ Hábito de fumar } \\
\hline Sim & 5 & 6,3 \\
\hline Não & 75 & 93,7 \\
\hline \multicolumn{3}{|l|}{ Uso de bebida alcoólica } \\
\hline Sim & 10 & 12,5 \\
\hline Não & 70 & 87,5 \\
\hline
\end{tabular}

a $S M=$ salário mínimo baseado no valor vigente em 2009 $(\mathrm{R} \$ 465,00)$.

TABELA 2. Consumo de nutrientes por puérperas de uma maternidade em Goiânia (GO), Brasil, 2008

\begin{tabular}{lccc}
\hline \multicolumn{1}{c}{ Nutriente } & Média \pm desvio padrão & Mediana & Valor de referência $^{a}$ \\
\hline Energia (Kcal) & $4611,54 \pm 1310,13$ & 4462,40 & Sem dados \\
Macronutrientes & $706,16 \pm 177,99$ & & \\
$\quad$ Carboidrato $(\mathrm{g})$ & $124,47 \pm 52,89$ & 703,38 & 175 \\
$\quad$ Lipídio $(\mathrm{g})$ & $165,79 \pm 58,99$ & 113,49 & Sem dados \\
$\quad$ Proteína $(\mathrm{g})$ & & 157,06 & 60 \\
Micronutrientes & $1314,33 \pm 631,58$ & 1119,27 & 1200 \\
$\quad$ Cálcio $(\mathrm{mg})$ & $24,57 \pm 8,79$ & 24,10 & 30 \\
Ferro $(\mathrm{mg})$ & $5784,87 \pm 7122,04$ & 2562,24 & 70 \\
Retinol $(\mu \mathrm{g})$ & $439,42 \pm 270,07$ & 388,51 & \\
Vitamina C $(\mathrm{mg})$ & $40,35 \pm 20,04$ & & Sem dados \\
Ácidos graxos & $71,56 \pm 29,33$ & 36,31 & Sem dados \\
Saturado $(\mathrm{g})$ & $521,72 \pm 382,75$ & 380,51 & Sem dados \\
Insaturado $(\mathrm{g})$ & & & \\
Colesterol $(\mathrm{mg})$ & & & \\
\hline
\end{tabular}

a Valores diários recomendados (12).

FIGURA 1. Valores de chumbo ( $\mu \mathrm{g} / \mathrm{L}$ ) em colostro de puérperas de uma maternidade em Goiânia (GO), Brasil, 2008

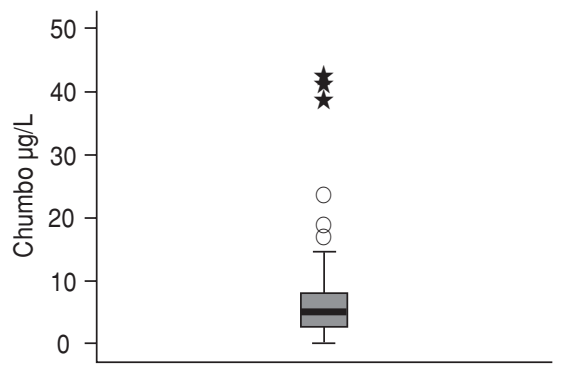

cidade caracterizada pela proximidade a vias de trânsito, rotas de lixo e diversos estabelecimentos industriais e comerciais (tabela 1).

Relativamente ao consumo alimentar, dentre os alimentos mais citados como parte da dieta, consumidos ao menos uma vez por dia, estiveram leite integral, bife bovino, arroz, pão francês, banana, laranja, alface, tomate, margarina, café e refresco industrial. Para esses alimentos foram obtidos os valores de energia, carboidratos, proteínas, lipídios e micronutrientes, conforme verificado na tabela 2. O consumo de praticamente todos esteve acima dos valores diários recomendados (11), exceto do metal ferro, com média um pouco abaixo do estabelecido.

A média da concentração de chumbo encontrada nas amostras de leite materno das puérperas estudadas foi de $6,88 \mu \mathrm{g} / \mathrm{L}$, com mediana de 4,65 $\mu \mathrm{g} / \mathrm{L}$ e variação de 0,12 a 41,5 $\mu \mathrm{g} / \mathrm{L}$. Três valores foram discrepantes dos demais (outliers), estando acima do terceiro quartil, como mostra a figura 1. Do total de amostras analisadas, 45,0\% apresen- taram valores acima de 5,0 $\mu \mathrm{g} / \mathrm{L}$ de chumbo - superiores ao segundo quartil (figura 1) representado pela mediana —, valor encontrado em estudos realizados pela Organização Mundial da Saúde (OMS) (12), que considerou uma faixa de 2,0 a 5,0 $\mu \mathrm{g} / \mathrm{L}$ em colostro humano.

Não houve associação entre os valores médios de chumbo nessas puérperas e as variáveis socioeconômicas, demográficas e de estilo de vida listadas na tabela 1. Tampouco houve associação entre os valores médios de chumbo e número de filhos e morar em região contaminada. $\mathrm{Na}$ correlação realizada entre o metal e o consumo alimentar, houve significância estatística para o valor de chumbo na amostra e o consumo de gordura saturada, colesterol e outros alimentos mencionados (tabela 3), demonstrando uma correlação entre o chumbo no colostro e o consumo dietético no período da gestação.

A regressão linear confirmou um efeito significativo sobre o chumbo encontrado no colostro das participantes deste estudo para as seguintes variáveis independentes: abóbora cozida; berinjela ensopada; produtos lácteos; ovos, carnes suínas e frango (tabela 4). A análise conjunta das variáveis independentes mostra que permaneceram relacionados ao teor do chumbo no colostro humano a abóbora cozida e os ovos, carne suína e frango.

\section{DISCUSSÃO}

Quase metade das amostras das participantes analisadas apresentou valores acima de 5,0 $\mu \mathrm{g} / \mathrm{L}$ para o chumbo. Esse resultado chama a atenção, pois esse foi o valor máximo obtido no colostro hu- 
TABELA 3. Correlação entre valores de chumbo $(\mu \mathrm{g} / \mathrm{L})$ nas amostras de colostro e variáveis socioeconômicas, de estilo de vida e consumo alimentar de puérperas de uma maternidade em Goiânia (GO), Brasil, 2008

\begin{tabular}{lrc}
\hline \multicolumn{1}{c}{ Variável } & \multicolumn{1}{c}{$\rho^{\mathrm{a}}$} & $P$ \\
\hline Características socioeconômicas & & \\
$\quad$ e clínicas & & \\
Idade (anos) & 0,135 & 0,135 \\
Tempo capital (anos) & $-0,166$ & 0,141 \\
$\quad$ Renda mensal (reais) & $-0,156$ & 0,166 \\
Macronutrientes & & \\
Energia (Kcal) & $-0,135$ & 0,231 \\
Carboidrato (g) & $-0,047$ & 0,682 \\
Lipídio (g) & $-0,198$ & 0,078 \\
Proteína (g) & $-0,184$ & 0,101 \\
Micronutrientes & & \\
Cálcio (mg) & $-0,220$ & 0,050 \\
Ferro (mg) & $-0,062$ & 0,583 \\
Retinol ( $\mu$ g) & 0,001 & 0,996 \\
Vitamina C (mg) & $-0,131$ & 0,247 \\
Ácidos graxos & & \\
Saturado (g) & $-0,260$ & 0,020 \\
Insaturado (g) & $-0,178$ & 0,114 \\
$\quad$ Colesterol (mg) & $-0,229$ & 0,041 \\
Alimentos & & \\
Abóbora cozida & 0,262 & 0,019 \\
Berinjela ensopada & 0,270 & 0,015 \\
Uva & $-0,243$ & 0,030 \\
Produtos lácteos (média) & $-0,229$ & 0,041 \\
Carnes bovinas (média) & $-0,245$ & 0,029 \\
Ovos, carnes suínas, frango & & \\
$\quad$ e derivados (média) & $-0,260$ & 0,020 \\
Massas e salgados (média) & $-0,239$ & 0,033 \\
\hline a Coeficiente de correlação de Spearman. & \\
& &
\end{tabular}

mano em estudos multicêntricos realizados pela OMS (12). Neste estudo, obtiveram-se três amostras de colostro com níveis acima de $35 \mu \mathrm{g} / \mathrm{L}$ de chumbo, podendo refletir uma exposição das puérperas a ambientes ou a alimentos contaminados com o metal. Essas mulheres receberam orientações orais, bem como panfletos e educação nutricional referentes à amamentação e à fase de $0 \mathrm{a}$ 2 anos de idade da criança.

Excetuando-se esses valores discrepantes, a maior parte das concentrações se encontra abaixo do nível considerado como fator de risco para a criança, que é acima de $20 \mu \mathrm{g} / \mathrm{L}$ (13). Isso indica que, nesta população, o colostro ingerido durante o aleitamento pelos recém-nascidos não é considerado, a princípio, nocivo e tóxico; porém, é preciso levar em conta o acúmulo ao longo do tempo no organismo.

Dois estudos no Brasil pesquisaram o teor de chumbo em leite maduro. O primeiro, realizado no Rio de Janeiro (14) com 38 amostras obtidas em população não exposta ao chumbo, obteve uma média de 2,8 $\mu \mathrm{g} / \mathrm{L}$ de chumbo, valor próximo aos 2,9 $\mu \mathrm{g} / \mathrm{L}$ observados pela pesquisa realizada em Londrina (15), cidade menos industrializada do que o Rio de Janeiro. Entretanto, os teores encontrados neste e nos estudos mencionados estão bem abaixo do teor encontrado em Taubaté, uma cidade bastante industrializada, próxima de São Paulo (1), onde o teor médio de chumbo encontrado foi de $154,4 \mu \mathrm{g} / \mathrm{L}$. Isso mostra que o teor de chumbo pode se relacionar à poluição ambiental advinda da industrialização, exposição ocupacional e maior povoamento, que consequentemente produz mais lixo urbano com resíduos tóxicos.

Mesmo referindo-se a uma região não muito industrializada, o teor médio de chumbo observado em nosso estudo, de $6,88 \mu \mathrm{g} / \mathrm{L}$, pode ser resultado de outras vias de exposição que não as ocupacionais, incluindo alimentos, ambiente e outras fontes que produzem exposição adicional - poeira e solo contaminados, pintura antiga de residências e ar contaminado por exaustão de automóveis e emissões industriais. As baterias armazenadas, não recicladas, de carros e eletrônicos em geral constituem também uma fonte importante de chumbo (16).

Na Áustria, pesquisadores encontraram níveis baixos de chumbo no leite materno maduro, de 1,63 $\mu \mathrm{g} / \mathrm{L}$, estando esses valores associados ao relato de fumo, consumo frequente de peixe e residência da mãe em cidades industrializadas (17). O estudo sugere ainda que o leite maduro possui menor concentração de chumbo do que o colostro, o que talvez explique o baixo teor obtido. Um estudo realizado na China encontrou média de chumbo de 7,68 $\mu \mathrm{g} / \mathrm{L}$ no colostro materno (18), valor aproximado ao deste estudo. No estudo chinês, os autores avaliaram também o leite maduro alguns dias depois do parto, obtendo um teor de chumbo menor $(2,34 \mu \mathrm{g} / \mathrm{L})$, o que indica que o nível do metal diminui com o tempo de lactação. Tal fato explica que os estudos realizados com colostro humano possam obter valores de chumbo mais elevados do que aqueles que utilizam apenas o leite maduro. $\mathrm{O}$ primeiro leite (colostro) possui maior conteúdo de elementos absorvidos durante a gestação do que o leite maduro $(14,15)$.

No presente estudo, as variáveis socioeconômicas, demográficas e de estilo de vida não se correlacionaram de forma significativa com o chumbo no colostro. Como em outros estudos $(1,15,19)$, a idade materna não refletiu um acúmulo de chumbo no organismo. As ocupações que as mães exerciam também não eram consideradas de risco $(6,7,14)$ para a exposição ao chumbo.

Considerando a alimentação, observamos uma correlação entre o chumbo e o consumo de berinjela e abóbora, o que mostra que os vegetais podem conter resíduos e contribuir para o teor de chumbo no colostro humano. $\mathrm{Na}$ regressão ajustada, a abóbora cozida foi um dos melhores preditores do chumbo no colostro. Com base nos resultados, pode-se dizer que o acréscimo de uma unidade de abóbora cozida implicou um aumento médio de 1,55 $\mu \mathrm{g} / \mathrm{L}$ no logaritmo do chumbo no colostro de puérpe-

TABELA 4. Variáveis independentes associadas aos valores de chumbo $(\mu \mathrm{g} / \mathrm{L})$ nas amostras de colostro em puérperas de uma maternidade em Goiânia (GO), Brasil, 2008

\begin{tabular}{|c|c|c|c|c|}
\hline \multirow[b]{2}{*}{ Variável } & \multicolumn{2}{|c|}{ Não ajustada } & \multicolumn{2}{|c|}{ Ajustada } \\
\hline & $\beta(\mathrm{ep})^{\mathrm{a}}$ & $P$ & $\beta(\text { ep })^{\mathrm{a}}$ & $P$ \\
\hline \multicolumn{5}{|l|}{ Alimentos } \\
\hline Abóbora cozida & $1,01(0,49)$ & 0,042 & $1,55(0,47)$ & 0,001 \\
\hline Berinjela ensopada & $3,57(1,8)$ & 0,049 & Sem dados & Sem dados \\
\hline Produtos lácteos (média) & $-1,07(0,53)$ & 0,046 & Sem dados & Sem dados \\
\hline Ovos, carnes suínas, frango (média) & $-3,37(1,71)$ & 0,049 & $-4,76(1,64)$ & 0,005 \\
\hline
\end{tabular}

a $\beta$ (ep) = coeficiente da regressão (erro padrão). 
ras, sendo um veículo desse resíduo na dieta das mulheres estudadas. Os processos de cocção não eliminam o contaminante chumbo, que é um elemento inorgânico que necessita de temperaturas extremas para modificar sua composição.

Um estudo de caso-controle (18) também verificou uma relação entre consumo de produtos de origem vegetal (ervas tradicionais chinesas) por mulheres e concentração de chumbo no leite materno. Nesse estudo, a média de chumbo das mulheres que consumiram as ervas foi de $8,59 \mu \mathrm{g} / \mathrm{L}$, nível mais elevado do que o encontrado no grupo de mães que não consumiram ervas tradicionais chinesas $(6,84 \mu \mathrm{g} / \mathrm{L})$. Isso faz sentido, visto que os metais pesados se acumulam frequentemente na camada superior do solo e na água que pode ser utilizada para irrigação. Atualmente, as principais fontes de chumbo no solo são constituídas de resíduos presentes nas imediações das estradas na forma de partículas de aerossóis e pó, além de cinzas de processos de fundição e de estruturas de demolição (20).

Os produtos de origem animal, como carne suína, leite e derivados, também demonstraram correlação significativa com o nível de chumbo obtido no colostro humano, todavia apresentaram coeficiente negativo. Como já descrito $(6,21)$, essa correlação negativa pode demonstrar menor absorção do chumbo. Isso se explica provavelmente pelo fato de tais produtos constituírem fonte de nutrientes essenciais (como proteínas, lipídios, cálcio, ferro) que, quando ingeridos adequadamente pelas gestantes, ou ainda acima do recomendado, tornam-se fatores antagônicos à absorção do contaminante chumbo. O efeito contrário foi confirmado na análise ajustada, em que o aumento de uma unidade de ovos, carnes e derivados consumidos pelas puérperas implicou decréscimo médio de 4,76 $\mu \mathrm{g} / \mathrm{L}$ no logaritmo do chumbo do colostro, reforçando a ideia de que esses alimentos podem conferir proteção ao organismo, reduzindo o acúmulo de chumbo.

Outros estudos $(5,22)$ mencionaram essa observação, encontrando correlação negativa entre dieta de cálcio e níveis de chumbo, utilizando, no entanto, o sangue como marcador biológico da pesquisa. Portanto, a baixa ingestão diária de cálcio contribui para o aumento de chumbo na corrente sanguínea (23); e o contrário também procede, como obser- vado neste estudo na análise ajustada, em que consumo maior de produtos ricos em cálcio indicou redução nos valores de chumbo encontrados no colostro materno das participantes.

Esses resultados reforçam a teoria de que um estado dietético adequado é importante e atua inversamente à absorção de contaminantes pelo organismo, como já relatado. Já foi mencionado anteriormente que a suplementação nutricional modifica o processo de absorção do chumbo, e a alimentação adequada com nutrientes essenciais pode reduzir ou até mesmo impedir a absorção desse metal (5). Isso respalda o motivo pelo qual os nutrientes ingeridos pelas puérperas deste estudo, por se encontrar acima do recomendado no caso dos macro e micronutrientes, exceto o retinol, podem ter redundado em correlações negativas, provavelmente sendo fator de proteção ao resíduo tóxico.

Dentre os nutrientes testados com o chumbo, ácido graxo saturado e colesterol apresentaram significância estatística com coeficientes negativos, indicando proteção contra o acúmulo de chumbo. Porém, vale ressaltar que o consumo elevado de alimentos ricos em lipídios e colesterol não é recomendável. Outro estudo realizado (24) com gordura saturada também obteve valor significativo $(P=$ $0,02)$, contudo utilizou amostras sanguíneas de crianças, ao contrário deste, que avaliou o colostro materno. Na pesquisa citada, observou-se correlação negativa significativa entre chumbo no sangue e carboidrato $(\mathrm{r}=-0,13)$ e vitamina $\mathrm{C}(\mathrm{r}=$ $0,14)$. Isso sugere a possibilidade de a vitamina $\mathrm{C}$ e o carboidrato serem fatores que agem inversamente à absorção do chumbo. A vitamina C é um antioxidante preventivo de intoxicação por chumbo, que reequilibra a oxidação prejudicada por esse metal, conforme descrito na literatura (25). Com isso, o maior consumo de uva pelas puérperas deste estudo, considerado produto antioxidante por conter vitamina $C$ em sua composição, além de outras propriedades, pode ter atuado como antagonista à quantidade de chumbo do colostro em nossa amostra.

Portanto, diante das evidências de que a puérpera pode transferir resíduos retidos em seu organismo ao filho na gestação, por meio da placenta, e na amamentação, por meio do leite, e que há vulnerabilidade maior na infância a elementos tóxicos, os níveis de chumbo no leite devem ser monitorados e controlados, inclusive por meio de uma alimentação materna equilibrada. Há relatos de três casos de intoxicação por chumbo em bebês (26) que estavam sendo amamentados, demonstrando que realmente o leite pode contaminar o neonato, dependendo da carga residual. É interessante que, no relato citado, os níveis de chumbo das mães foram sempre mais altos no leite materno do que no sangue (26). Assim, confirma-se que o leite materno e, em especial, o colostro (marcador biológico utilizado neste estudo), é um método eficiente para estimar a concentração de chumbo da puérpera durante o aleitamento. Além disso, o uso do colostro ou leite materno não é invasivo e não traz risco, retratando a concentração real dos teores de resíduos presentes.

Apesar de ser um método eficiente para estimar o conteúdo de metais, o teor de gordura do leite, mais elevado no colostro, é fator complicador para a determinação de chumbo na matriz biológica $(27,28)$, exigindo mais cuidado no preparo de amostras para garantir sensibilidade na detecção do chumbo. Observouse dificuldade em estabelecer um nível de referência para a concentração de chumbo em leite e colostro humano, porque as variações entre os estudos publicados sobre o tema refletem inúmeras condições que podem interferir no resultado final, como, por exemplo, metodologias analíticas diversas.

Conclui-se que as características maternas e ambientais podem ter levado à presença do chumbo no colostro humano das participantes desta pesquisa. Principalmente, parece que as práticas dietéticas agiram como fator protetor ou de risco para a absorção de contaminantes. Todas as medidas possíveis devem ser tomadas para reduzir a contaminação ambiental, materna e dos lactentes pelo chumbo, por meio da adoção de hábitos saudáveis de vida e alimentares, com orientação de profissionais no momento do pré-natal para uma educação nutricional adequada.

Mesmo que as crianças filhas das puérperas deste estudo não estejam ameaçadas de intoxicação grave pelo chumbo ingerido no colostro, percebe-se a necessidade de atenção especial nessa fase do desenvolvimento, em que o organismo se encontra mais suscetível. Ressalta-se que os benefícios oferecidos pelo aleitamento materno à criança estão 
além da biodisponibilidade do chumbo. Por isso, a amamentação nos primeiros 6 meses de vida deve ser sempre estimulada, a não ser que a exposição materna seja muito grande, a ponto de causar efeitos orgânicos tóxicos.

Como os resultados do presente estudo se referem especificamente às puérperas de uma maternidade de Goiânia, é recomendável a realização de novas pesquisas sobre os valores desse resíduo no leite materno, não só regionalmente, como em todo o País. Especialmente devido à crescente poluição ambiental, é fundamental conhecer as principais fontes de chumbo diante de diversos ambientes e estilos de vida, assim como o seu impacto na saúde dos seres vivos.
Agradecimentos. Os autores agradecem ao laboratório do Ministério da Agricultura, Pecuária e Abastecimento, pelo apoio logístico. RMG recebeu bolsa de doutorado da Coordenação de Aperfeiçoamento de Pessoal de Nível Superior (CAPES).

\section{REFERÊNCIAS}

1. Nascimento LFC, Izário Filho HJ, Baltazar EO. Níveis de chumbo em colostro humano: um estudo no Vale do Paraíba. Rev Bras Saude Mater Infant. 2006;6(1):69-74.

2. Agency for Toxic Substances and Disease Registry. Toxicological profile for lead. Washington, D.C.: U.S. Department of Health and Human Services; 2005. Disponível em: www.atsdr.cdc.gov/toxprofiles/tp13.pdf. Acessado em 23 de novembro de 2009.

3. Soldin OP, Hanak B, Soldin SJ. Blood lead concentration in children: new range. Clin Chim Acta. 2003;327(1-2):109-13.

4. Lidsky TI, Schneider JS. Lead neurotoxicity in children: basic mechanisms and clinical correlates. Brain. 2003;126(Pt 1):5-19.

5. Dorea JG, Donangelo CM. Early (in uterus and infants) exposure to mercury and lead. Clin Nutr. 2006;25(3):369-76.

6. Gonçalves JR, Mesquita AJ, Gonçalves RM. Determinação de metais pesados em leite integral bovino pasteurizado no Estado de Goiás. Cienc Anim Bras. 2008;9(2):365-74.

7. Paoliello MMB, De Capitani EM. Occupational and environmental human lead exposure in Brazil. Environ Res. 2007;103(2): 288-97.

8. Fornés NS, Stringhini MLF. Development of a food frequency questionnaire (FFQ) and characterization of the food pattern consumption for low-income workers in the city of Goiânia, Goiás State, Brazil. Acta Sci Health Sci. 2005;26(1):69-75.

9. Fornés NS, Stringhini MLF, Elias BM. Reproducibility and validity of a food-frequency questionnaire for use among low-income Brazilian workers. Public Health Nutr. 2003; 6(8):821-827.

10. Brasil, Ministério da Saúde, Agência Nacional de Vigilância Sanitária, Instituto Adolfo Lutz. Métodos físico-químicos para análise de alimentos. Brasília: Ministério da Saúde; 2005.

11 National Research Council. Recommended dietary allowances. 10th ed. Washington, D.C.: National Academy Press; 1989.
12. World Health Organization, International Atomic Energy Agency. Minor and trace elements in breast milk: report of a joint WHO/IAEA collaborative study. Genebra: WHO/IAEA; 1989.

13. World Health Organization. Trace elements in human nutrition and health. Genebra: WHO; 1996.

14. Anastacio AS, Silveira CLP, Miekeley N, Donangelo CM. Distribution of lead in human milk fractions: relationship with essential minerals and maternal blood lead. Biol Trace Elem Res. 2004;102(1-3):27-37.

15. Koyashiki GAK. Níveis de chumbo em leite e sangue de doadoras de banco de leite em município do sul do Brasil [dissertação]. Londrina: Universidade Estadual de Londrina; 2008.

16. Mattos RCOC, Carvalho MAR, Mainenti HRD, Xavier Junior EC, Sarcinelli PN, Carvalho LBV, et al. Avaliação dos fatores de risco relacionados à exposição ao chumbo em crianças e adolescentes do Rio de Janeiro. Cienc Saude Coletiva. 2009;14(6):2039-48.

17. Gundacker C, Pietschnig B, Wittmann KJ, Lischka A, Salzer H, Hohenauer L, et al. Lead and mercury in breast milk. Pediatrics. 2002;110:873-8

18. Chien L, Yeh C, Lee H, Chao HJ, Shieh MJ, Han BC. Effect of the mother's consumption of traditional Chinese herbs on estimated infant daily intake of lead from breast milk. Sci Total Environ. 2006;354(2-3):120-6.

19. Ettinger AS, Téllez-Rojo MM, Amarasiriwardena C, Gonzalez-Cossio T, Peterson KE, Aro $\mathrm{H}$, et al. Levels of lead in breast milk and their relation to maternal blood and bone lead levels at one month postpartum. Environ Health Perspect. 2004;112(8):926-31

20. de Freitas CU, De Capitani EM, Gouveia N, Simonetti MH, Paula e Silva MR, Kira CS, et al. Lead exposure in an urban community: investigation of risks factors and assessment of the impact of lead abatement measures. Environ Res. 2007;103(3):338-44
21. Gonçalves JR. Determinação de $\mathrm{Pb}, \mathrm{Cd}, \mathrm{Fe}$, $\mathrm{Zn}$ e Cu em carnes de bovinos e $\mathrm{Pb}, \mathrm{Cd}$ e $\mathrm{P}$ em suplementos minerais no estado de Goiás [tese]. Goiânia: Universidade Federal de Goiás; 2007.

22. Ballew C, Khan LK, Kaufmann R, Mokdad A, Miller DT, Gunter EW. Blood lead concentration and children's anthropometric dimensions in the Third National Health and $\mathrm{Nu}-$ trition Examination Survey (Nhanes III), 1988-1994. J Pediatr. 1999;134(5):623-30.

23. Gulson BL, Mizon KJ, Korsch MJ, Palmer JM, Donnelly JB. Mobilization of lead from human bone tissue during pregnancy and lactation: a summary of long-term research. Sci Total Environ. 2003;303(102):79-104.

24. Gallicchio L, Scherer RW, Sexton M. Influence of nutrient intake on blood lead levels of young children at risk for lead poisoning. Environ Health Perspect. 2002;110(12):A767-72.

25. Ahamed M, Siddiqui MKJ. Environmental lead toxicity and nutritional factors. Clin Nutr. 2007;26(4):400-8.

26. Godinho AF, Vassilief I, Silva AG, Aguiar DC. Intoxicação por chumbo em bebês lactentes em três casos de exposição maternal. Salusvita. 2001;20(3):11-8.

27. Needham LA, Wang RY. Analytic considerations for measuring environmental chemicals in breast milk. Environ Health Perspect. 2002;110(6):317-24.

28. Ettinger AS, Téllez-Rojo MM, Amarasiriwardena C, Bellinger D, Peterson K, Schwartz J, et al. Effect of breast milk lead on infant blood lead levels at 1 month of age. Environ Health Perspect. 2004;112(1):1381-5.

Manuscrito recebido em 27 de maio de 2010. Aceito em versão revisada em 26 de outubro de 2010. 
ABSTRACT Objective. To estimate the levels of lead in human colostrum and investigate whether they are correlated with dietary intake and socioeconomic factors in puerperal women.

Relationship between lead levels in colostrum, dietary intake, and socioeconomic characteristics of puerperal women in Goiânia, Brazil
Method. This cross-sectional study was carried out with 80 puerperal women from a maternity hospital in Goiânia, Brazil. Data were collected between July and December 2008. A questionnaire was used to collect socioeconomic and lifestyle information. A dietary intake frequency questionnaire was also applied. After that, colostrum samples were collected and analyzed using flame atomic absorption spectrometry to quantify lead levels. The correlation between lead levels, socioeconomic variables, and dietary intake was calculated.

Results. The mean concentration of lead in colostrum samples was $6.88 \mu \mathrm{g} / \mathrm{L}$, and the median concentration was $4.65 \mu \mathrm{g} / \mathrm{L}$. There was no association between socioeconomic aspects and lead levels. The intake of saturated fatty acids, eggs, pork meats, chicken, and chicken products $(r=-0.26$ and $P=0.020)$ was inversely correlated with the levels of lead in human colostrum, whereas the intake of squash $(\mathrm{r}=$ $0.26 ; P=0.019)$ and eggplant $(r=0.27 ; P=0.015)$ was positively correlated with the levels of lead. The correlation observed for cooked squash, eggs, pork and chicken meats, and chicken products was maintained after logistic regression adjustment. Conclusions. The presence of lead was detected in human colostrum in this sample, probably as a result of dietary exposure. In addition to a correlation between presence of lead and dietary intake, the present findings suggest that specific nutrients may result in increased or decreased levels of lead, without a direct relationship with socioeconomic factors.

Key words Lead; food consumption; colostrum; milk, human; toxicity; Brazil. 